\title{
Effects of mandibular advancement on respiratory resistance
}

\author{
A.M. Lorino*, M. Maza ${ }^{\S}$, M.P. d'Ortho*, A. Coste ${ }^{\#}$ A. Harf*, H. Lorino*
}

Effects of mandibular advancement on respiratory resistance. A.M. Lorino, M. Maza, M.P. d'Ortho, A. Coste, A. Harf, H. Lorino. C)ERS Journals Ltd 2000.

ABSTRACT: Mandibular advancing devices are proposed as nonsurgical treatment for certain patients with an obstructive sleep apnoea syndrome. Since they act by increasing the upper airway calibre, the aim of the present study was to investigate the changes in respiratory resistance $(R \mathrm{rs})$ resulting from mandibular advancement.

$R$ rs was measured at the nose by the forced oscillation technique $(4-32 \mathrm{~Hz})$. Ten normal subjects were studied under three conditions: resting mandibular position, passive mandibular advancement steadied by a wax bite, and voluntary advancement, in random order. Respiratory resistance was extrapolated to $0 \mathrm{~Hz}(R 0)$ and estimated at $16 \mathrm{~Hz}(R 16)$ by linear regression analysis of respiratory resistive impedance versus frequency.

$R 0\left(\right.$ mean $\pm \mathrm{SEM}=3.5 \pm 0.2 \mathrm{cmH}_{2} \mathrm{O} \cdot \mathrm{L}^{-1} \cdot \mathrm{s}$ in the resting position) decreased significantly with passive advancement $\left(2.9 \pm 0.2 \mathrm{cmH}_{2} \mathrm{O} \cdot \mathrm{L}^{-1} \cdot \mathrm{s}, \mathrm{p}<0.001\right)$, but remained unchanged with voluntary mandibular advancement $\left(3.6 \pm 0.2 \mathrm{cmH}_{2} \mathrm{O} \cdot \mathrm{L}^{-1} \cdot \mathrm{s}\right)$. Similar results were obtained for $R 16$.

The results of this study demonstrate that the effects of mandibular advancement on upper airway resistance differ, depending on whether advancement is passive or active, and suggest that in order to simulate the actual effects of therapeutic devices, mandibular advancement should be passive.

Eur Respir J 2000; 16: 928-932.
*INSERM U 492 et Service de Phy-

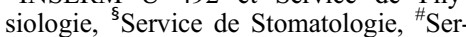
vice d'Oto-rhino-laryngologie, Hôpital Henri Mondor, AP-HP, et Université Paris X11, 94000 Créteil, France.

Correspondence: A.M. Lorino, Service de Physiologie - Explorations Fonctionnelles, Hôpital Henri Mondor, 94010 Créteil, France. Fax: 33149812698

Keywords: Forced oscillation mandibular advancement respiratory resistive impedance upper airway calibre

Received: March 62000 Accepted after revision June 232000
Obstructive sleep apnoea-hypopnoea syndrome (OSAS) is characterized by repetitive upper airway narrowing and/ or closure during sleep. Obstructive respiratory events are generally explained by the inefficiency of the dilating muscles in counterbalancing the tendency of the pharyngeal airway to collapse, due to the inspiratory decrease in transmural pressure [1], and one of the main factors predisposing subjects to OSAS appears to be a reduction in upper airway calibre [2].

Several types of treatment are currently used in OSAS patients. The most widely used is nasal continuous positive airway pressure (nCPAP), which prevents the upper airway from being subjected to a negative intraluminal pressure during inspiration. However, some patients refuse nCPAP treatment straight away, and others, in whom nCPAP has been initiated, discontinue it because they do not tolerate it. An alternative treatment which is becoming widespread [3, 4], is a dental orthosis, which induces temporary mandibular advancement, and thereby increases the upper airway space. Studies based on cephalometric measurements or videoendoscopic images have shown that both passive and voluntary mandibular advancement increases various upper airway dimensions in awake patients. In particular, the following have been reported: 1) an increase in the superior airway space located between the soft palate and the posterior nasopharynx [5];2) an increase [6], or no change $[5,7]$, in the posterior airway space, whereas a marked increase in posterior airway space was observed with maximal mandibular advancement [8]; 3) a decrease in the mandible-hyoid distance [7]; and 4) an increase in the cross-sectional area of the hypopharynx and oropharynx with maximal voluntary advancement [9]. However, it is likely that orthosisinduced changes in upper airway geometry vary with the type of device used, and for a given orthosis, from one patient to another.

Such studies, which are based on cephalometric radiographs or on videoendoscopic images, provide only geometrical descriptions of the upper airway and do not give information on the effect of mandibular advancement on resistive properties of the upper airway during spontaneous breathing. The authors reasoned that upper airway resistance might be a good mechanical index of the effects of mandibular advancement, since it reflects the overall result of changes at different anatomical levels of the airway. The purpose of the present study was therefore, to investigate the changes in upper airway resistance induced by mandibular advancement, as deduced from those in respiratory resistance $(R \mathrm{rs})$. The authors compared the respective influences on upper airway resistance of voluntary and passive mandibular advancement, since the former has the advantage of requiring no particular device. The forced oscillation technique which allows easy measurement of $R$ rs during spontaneous nasal breathing was used.

\section{Materials and methods}

Subjects

The authors studied 10 asymptomatic healthy subjects (6 males and 4 females), aged 28-57 yrs, with no upper or 
lower respiratory complaints. None of the subjects were habitual snorers, or complained of the diurnal or nocturnal symptoms of the sleep apnoea syndrome. Only 2 were moderate smokers ( 5 cigarettes $\cdot$ day $\left.^{-1}\right)$.

\section{Respiratory resistance measurement}

Respiratory impedance ( $\mathrm{rrs}$ ) was measured by forced oscillation during spontaneous nasal breathing with the mouth closed. Subjects were seated comfortably in a chair, facing a table, and asked to breathe quietly through the nose while keeping the mouth closed. They supported their cheeks with their hands, the elbows resting on the table in order to allow a reproducible position to be maintained during each measurement. The pseudo-random forced flow was applied at the nose via a rigid nasal mask. It was composed of 29 harmonics $(4-32 \mathrm{~Hz})$ of the fundamental $(1 \mathrm{~Hz})$, with enhanced amplitudes at the lower frequencies to limit the influence of spontaneous breathing. The phases were calculated in order to minimize the peak-to-peak amplitude of the excitation signal. The forced signal, generated by a digital-to-analogue converter, excited, through a power amplifier, a 50-W loudspeaker (Audax HM 130 XO Château du Loir, France).

The peak-to-peak amplitude of the resulting flow was about $0.2 \mathrm{~L} \cdot \mathrm{s}^{-1}$. Nasal flow $\left(V^{\prime}\right)$ was measured with a screen pneumotachograph (resistance $=0.35 \mathrm{cmH}_{2} \mathrm{O} \cdot \mathrm{L}^{-1} \cdot \mathrm{s}$; Jaeger, Wurzburg, Germany) connected to a differential pressure transducer (Sensym SCX 01D, $\pm 70 \mathrm{cmH}_{2} \mathrm{O}$, Sunnyvale, CA, USA), and nasal pressure (P), by a similar pressure transducer referenced to the atmosphere. The pneumotachograph and tubing were flushed by a constant bias flow $\left(0.5 \mathrm{~L} \cdot \mathrm{s}^{-1}\right)$. Pressure and flow data were collected over $16 \mathrm{~s}$ periods and high-pass filtered (3rd order, cut-off frequency $=3.5 \mathrm{~Hz}$ ) to eliminate the low harmonics of the breathing noise. A fast Fourier transform algorithm was applied to adjacent $4 \mathrm{~s}$ periods. Impedance data were calculated from the auto- and cross-spectra of $\mathrm{P}$ and $V^{r}$ obtained by averaging the spectra of 3-4 consecutive manoeuvres, and retained for analysis when they showed a coherence value $>0.9[10]$. Respiratory resistance $(R \mathrm{rs})$ was submitted to linear regression analysis versus frequency over the 4-16 and 16-32 Hz frequency range. $R \mathrm{rs}$ extrapolated to $0 \mathrm{~Hz}(R 0)$ was derived from the first linear regression analysis, and $R \mathrm{rs}$, estimated at $16 \mathrm{~Hz}(R 16)$, from the second linear regression analysis [11].

For each data set, respiratory frequency, tidal volume, and maximal inspiratory flow, were measured from the flow signal, after low-pass filtering.

\section{Experimental protocol}

Each subject was studied under three conditions: resting mandibular position, passive mandibular advancement steadied by a wax bite designed at the subjects' convenience and resulting in a forward mandible advancement of about 5-6 $\mathrm{mm}$ against the rest position, and voluntary mandibular advancement. The order of the two last conditions was selected randomly, and a 3-min period was allowed before each set of measurements with mandibular advancement.

\section{Data analysis}

Values are expressed as mean \pm SEM, except when otherwise indicated. Statistical analysis of data was performed using repeated-measure analysis of variance, completed as necessary by a paired t-test. A value of $\mathrm{p}<0.05$ was considered significant.

\section{Results}

Typical respiratory resistance curves obtained in a representative subject are shown in figure 1. No significant changes in either respiratory frequency, tidal volume, or maximal inspiratory flow, were observed between the resting position and mandibular advancement (table 1).

In the resting mandibular position, $R 0$ and $R 16$ were $3.5 \pm$ $0.2 \mathrm{cmH}_{2} \mathrm{O} \cdot \mathrm{L}^{-1} \cdot \mathrm{s}$ and $3.9 \pm 0.2 \mathrm{cmH}_{2} \mathrm{O} \cdot \mathrm{L}^{-1} \cdot \mathrm{s}$, respectively.

As shown in figure $2, R 0$ decreased significantly with passive advancement $\left(2.9 \pm 0.2 \mathrm{cmH}_{2} \mathrm{O} \cdot \mathrm{L}^{-1} \cdot \mathrm{s}, \mathrm{p}<0.001\right)$, whereas it did not change with voluntary advancement $\left(3.6 \pm 0.2 \mathrm{cmH}_{2} \mathrm{O} \cdot \mathrm{L}^{-1} \cdot \mathrm{s}\right)$, due to the wide intersubject variability (fig. 3). Similarly, $R 16$ decreased significantly with passive advancement $\left(3.3 \pm 0.2 \mathrm{cmH}_{2} \mathrm{O} \cdot \mathrm{L}^{-1} \cdot \mathrm{s}, \mathrm{p}<0.01\right)$, but did not change with voluntary advancement (4.0 \pm 0.2 $\left.\mathrm{cmH}_{2} \mathrm{O} \cdot \mathrm{L}^{-1} \cdot \mathrm{s}\right)$. No significant difference was observed between the changes in $R 0$ and $R 16$ induced by passive advancement.

\section{Discussion}

Since mandibular advancement devices are proposed as nonsurgical treatment for certain patients with OSAS, the effects of mandibular advancement on upper airway geometry have been widely investigated. Until now, these effects have been assessed by anatomical parameters derived from cephalometric radiographs or videoendoscopic images, and not by mechanical parameters based on physiological measurements. Furthermore, to the authors' knowledge, no distinction has ever been drawn between passive and active mandibular advancement. Therefore, it

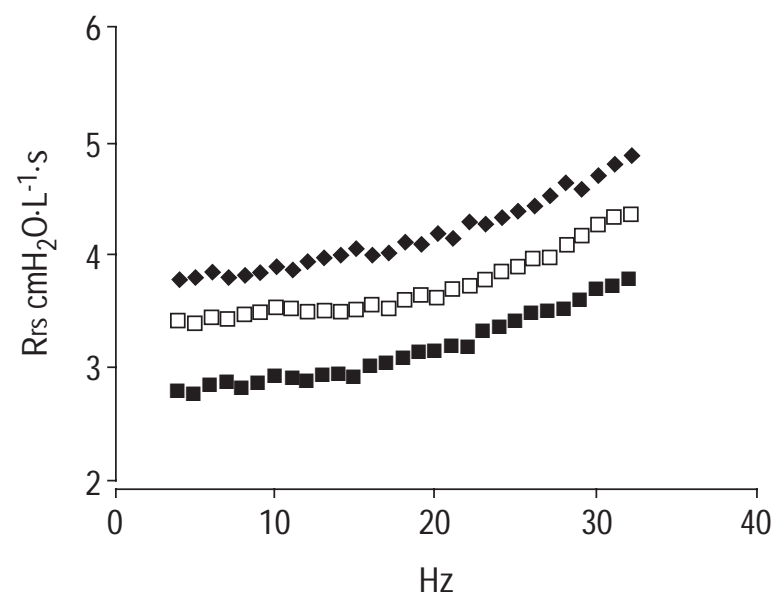

Fig. 1. - Respiratory resistance $(R \mathrm{rs})$ in a representative subject with mandibular rest position (MRP), passive mandibular advancement (PMA), and voluntary mandibular advancement (VMA). $\downarrow$ : VMA; $\square$ : MRP; $\square$ : PMA. 
Table 1. - Ventilatory parameters in the three mandibular positions

\begin{tabular}{llll}
\hline & \multicolumn{1}{c}{ MRP } & \multicolumn{1}{c}{ PMA } & \multicolumn{1}{c}{ VMA } \\
\hline RF min $^{-1}$ & $14.2 \pm 1.2$ & $13.9 \pm 1.1$ & $14.0 \pm 1.3$ \\
VT L & $0.62 \pm 0.07$ & $0.57 \pm 0.07$ & $0.64 \pm 0.06$ \\
$V^{\prime}$ maxi L $\cdot s^{-1}$ & $0.47 \pm 0.03$ & $0.45 \pm 0.03$ & $0.49 \pm 0.03$
\end{tabular}

Data are presented as mean \pm SEM $(n=10)$. MRP: mandibular rest position; PMA: passive mandibular advancement; VMA: voluntary mandibular advancement; RF: respiratory frequency; VT: tidal volume; Vmaxi: maximal inspiratory flow.

appeared interesting to investigate the influence of both types of mandibular advancement on airway resistance, which takes into account the dynamic changes in upper airway geometry induced by ventilation, hence reflecting the actual resistive load. The results demonstrate that airway resistance clearly decreases with passive mandibular advancement, but remains unchanged with voluntary advancement.

The authors chose the forced oscillation technique because it allows noninvasive measurement of respiratory resistance, and is the only method which is easily applicable when the mouth is closed. Furthermore, Rrs has been shown to detect variations in upper airway mechanics resulting from postural changes [12]. From a theoretical point of view, the use of forced oscillation applied at the nose might appear debatable due to the flow-dependence of nasal resistance. $Z$ rs measurement with this method is based on the assumption that the mechanical system considered behaves linearly. However, small flow oscillations are expected to linearise the mechanical behaviour of the respiratory system including the nose. It has been observed that the nonlinear characteristics of the transnasal pressure-flow relationship, which are pronounced at $1-2 \mathrm{~Hz}$, diminish as the frequency increases [13]. It has also been shown that nasal resistance can be assessed by forced oscillation [14], and that respiratory resistance, measured at the nose during continuous positive airway pressure titration, reflects lung resistance determined from transpulmonary pressure [15].

It would have been more relevant to the clinical situation if the measurements had been made in the supine position.

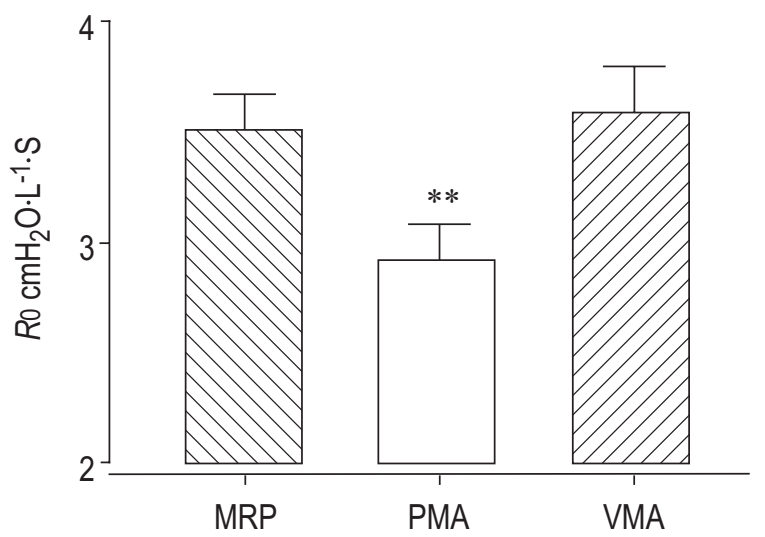

Fig. 2. - Mean values $(\mathrm{n}=10)$ of respiratory resistance extrapolated to 0 $\mathrm{Hz}(R 0)$ with mandibular rest position (MRP), passive mandibular advancement (PMA), and voluntary mandibular advancement (VMA). Bars indicate SE. **: significantly lower than with MRP $(\mathrm{p}<0.001)$.

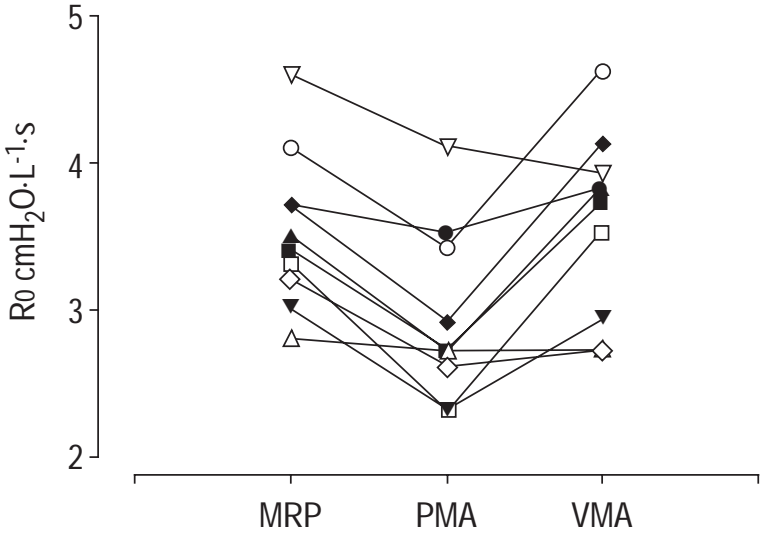

Fig. 3. - Individual values for respiratory resistance extrapolated to 0 $\mathrm{Hz}(R 0)$ in the 10 subjects, with mandibular rest position (MRP), passive mandibular advancement (PMA), and voluntary mandibular advancement (VMA).

However, the authors chose the sitting position for this preliminary study because it was previously observed, in the course of rhinomanometric studies, that nasal resistance values are unstable in the supine position. In this study in which Rrs was measured at the nose and thereby took into account nasal resistance, it appeared important to exclude the influence of nasal congestion, with the aim of more accurately assessing the effect of mandibular advancement on airway resistance.

Care was taken to keep the same position under all three conditions, because neck extension and/or flexion has been reported to affect upper airway geometry, and consequently $R \mathrm{rs}$ [16]. As the ventilatory flow profile was similar under all three conditions (table 1), any change in $R$ rs could be assumed to reflect the change in upper airway resistance, and therefore, to be attributable to the effect of mandibular advancement.

Two indices of respiratory resistance were chosen, namely $R 16$ which represents Newtonian respiratory resistance, and $R 0$ which reflects total respiratory resistance, i.e. Newtonian resistance plus the additional airflow resistance resulting from series or parallel gas redistribution, when present [11]. $R 0$ and $R 16$ responded similarly to passive advancement demonstrating that the effect of mandibular advancement originates from changes in instantaneous respiratory resistance, i.e. in upper airway resistance.

As expected, basal $R 0$ and $R 16$ values, which took into account nasal resistance, were higher than those generally reported for respiratory resistances measured at the mouth, i.e. with the nasal pathway shunted [12]. The small but significant difference observed between $R 0$ and $R 16$ $(\mathrm{p}<0.002)$ reflects the slight positive frequency dependence of $R$ rs often observed in subjects with normal lungs.

Passive advancement resulted in significant and similar decreases in $R 0$ and $R 16$, a result which is in accordance with the changes in upper airway geometry previously reported with orthoses, namely a marked increase in the superior airway space [5], a decrease in the mandiblehyoid distance [7], and an increase in the posterior airway space [6], although the latter result has not always been found $[5,7]$.

Surprisingly, when compared to the resting mandibular position, voluntary advancement did not induce significant 
changes in $R 0$ or $R 16, R$ rs increased in six subjects, and decreased in the other four (fig. 3). It might be assumed that, passive or voluntary, mandibular advancement would induce decreases in $R 0$ or $R 16$, since both types of advancement have been reported to induce increases in the superior airway space. Several investigators have previously used voluntary advancement to simulate the effects of orthoses on upper airway geometry $[8,9]$. The difference presently observed between the Rrs responses to passive and voluntary advancement cannot be attributed to an effect of lung volume on intrathoracic airways. Functional residual capacity (FRC) for the three mandibular conditions in the three subjects who had the greatest passive advancement-induced decreases and voluntary advancement-induced increases in $R$ rs were measured. As no significant difference was found between the FRC values obtained in the three conditions, it may be assumed that intrathoracic airway resistance remained unchanged, and that the changes observed in Rrs could be explained by those in upper airway resistance only. Consequently, the results clearly demonstrate that: 1) the mechanical factors which determine upper airway resistance are different during passive and voluntary advancement in most subjects; 2) voluntary advancement cannot be used to simulate the effect of orthoses on upper airway resistance; and 3) as previously suggested [7], the mechanisms involved in mandibular advancement do not amount to simple increases in airway calibre.

The experimental conditions did not allow for the measurement of the extent of voluntary mandibular advancement, but it was observed that protrusion was more pronounced with voluntary than with passive advancement. Cephalometric studies in OSAS patients have shown a $56 \%$ mean increase in posterior airway space when maximal active mandibular protrusion was compared to the rest position [8]. In addition, videoendoscopic investigations in OSAS patients and nonapnoeic control subjects have demonstrated that both groups respond similarly to maximal voluntary advancement by increasing hypopharynx and oropharynx cross-sectional areas [9]. Furthermore, three dimensional videoendoscopic images have shown that mandibular protrusion results in a change in the shape of the upper airway from a laterally oriented ellipse to a somewhat more circular contour [9], which tends to reduce friction forces and further decrease upper airway resistance.

So how can the difference presently observed between passive and voluntary advancements, and the apparent discrepancy between anatomical and mechanical measurements be explained?

A possible explanation for the results might be the difference between upper airway geometry and mechanics, which suggests that upper airway resistance, which is a dynamic measurement, cannot be predicted from a static anatomical description of the airway. Cephalometric radiographs are snapshots, and measurements from videoendoscopic images have been made at end-tidal expiration, i.e. when respiratory muscles are inactive. Consequently, the structural changes in the upper airway induced by ventilation, such as those resulting from the aperture of the glottis, movement of soft tissues at the pharyngeal and/or palatal levels, and contraction of the upper airway dilating muscles, were not taken into account. These changes might differ with passive and voluntary protrusion, since an increase has been reported in baseline genioglossus activity in cats after insertion of a bite block [17]. Voluntary advancement activates both the dilatator and constrictor muscles of the upper airway. Therefore, with voluntary protrusion, the beneficial effect of the anterior tongue displacement might be counteracted by the concomitant activation of upper airway constrictor muscles. The level of activation of these muscles was probably related to the mandibular effort, and thereby variable between subjects, since some of them reported slight pain in the temporomandibular joint during voluntary protrusion. This might explain the intersubject variability of the $R$ rs response.

Another explanation for the results, and probably for the fact that no consistent correlation has been found between individual changes in the posterior airway space and degrees of improvement with orthoses in OSAS patients [7], might be the role played by those parts of the upper airway which are explored by neither cephalometry or videoendoscopy, namely the nasal and laryngeal regions. The $R$ rs indices took into account total airway resistance, and therefore, nasal and laryngeal resistance, which may be influenced by the nature of mandibular advancement. A significant increase in nasal resistance has been observed in response to caudal tracheal displacement in paralyzed rats in whom the upper airway was isolated [18], and changes in nasal patency are among the stimuli that activate afferent nerves of the larynx [19].

Whatever the mechanisms involved in upper airway resistance, the results suggest that the effect of mandibular advancement probably depends on the level of muscular contraction, and that total upper airway resistance should be taken into account in the prospective studies aiming at predicting the efficiency of mandibular advancement devices.

To conclude, cephalometric and videoendoscopic investigations on the one hand, and physiological investigations on the other, appear to provide different information about the influence of mandibular advancement on the upper airway. The results demonstrate that the effects of mandibular advancement on upper airway resistance differ, depending on whether advancement is passive or active, and that to simulate the actual effects of mandibular advancement devices, nasal resistance should be taken into account, and mandibular advancement should be passive. Further studies performed on nonapnoeic heavy snorers and obstructive sleep apnoea-hypopnoea syndrome patients, including polysomnographic investigations, are still required to evaluate the ability of the respiratory resistance response to passive advancement to predict the efficacy of mandibular advancement in treating sleep respiratory events.

Acknowledgements. The authors wish to thank F. Lofaso for helpful reviewing of the manuscript.

\section{References}

1. Deegan PC, McNicholas WT. Pathophysiology of obstructive sleep apnoea. Eur Respir Mon 1998; 10: 29-62.

2. Bradley TD, Brown IG, Grossman RF, et al. Pharyngeal size in snorers, nonsnorers, and patients with obstructive sleep apnea. $N$ Engl J Med 1986; 315: 1327-1331. 
3. Lowe AA. Dental appliances for the treatment of snoring and obstructive sleep apnea. In: Kryger M, Roth T, Dement W, eds. Principle and Practice of Sleep Medicine. Philadelphia, Saunders, 1994; pp. 722-735.

4. Schmidt-Nowora W, Lowe A, Wiegand L, Cartwright R, Perez-Guerra F, Menn S. Oral appliances for the treatment of snoring and obstructive sleep apnea. Sleep 1995; 18: 501-510.

5. Bonham PE, Currier GF, Orr WC, Othman J, Nanda RS. The effect of a modified functional appliance on obstructive sleep apnea. Am J Orthond Dentofacial Orthop 1988; 94: 384-392.

6. Schmidt-Nowra WW, Meade TE, Hays MB. Treatment of snoring and obstructive sleep apnea with a dental orthosis. Chest 1991; 99: 1378-1385.

7. Eveloff SE, Rosenberg CL, Carlish CC, Millman RP. Efficacy of a Herbst mandibular advancement device in obstructive sleep apnea. Am J Respir Crit Care Med 1994; 148: 905-909.

8. Johnson LM, Arnett GW, Tamborello JA, Binder A. Airway changes in relationship to mandibular posturing. Otolaryngol Head Neck Surg 1992; 106: 143-148.

9. Ferguson KA, Love LL, Ryan CF. Effect of mandibular and tongue protusion on upper airway size during wakefulness. Am J Respir Crit Care Med 1997; 155: 17481754.

10. Lorino H, Mariette C, Karouia M, Lorino AM. Influence of signal processing on estimation of respiratory impedance. J Appl Physiol 1993; 74: 215-223.

11. Lorino AM, Zerah F, Mariette C, Harf A, Lorino H. Respiratory resistive impedance in obstructive patients: linear regression analysis versus viscoelastic modelling. Eur Respir J 1997; 10: 150-155.
12. Lorino AM, Atlan G, Lorino H, Zanditenas D, Harf A. Influence of posture on mechanical parameters derived from respiratory impedance. Eur Respir J 1992; 5: 11181122.

13. Sullivan KJ, Chang HK. Flow dynamics of the nasal passage. In: Farrel Epstein MA, Ligas JR, eds. Respiratory Biomechanics, Engineering Analysis of Stucture and Function. New York, Sprinler-Verlag, 1990; pp. 98105.

14. Lorino AM, Lofaso F, Abi-Nader F, et al. Nasal airflow resistance measurement: forced oscillation technique versus posterior rhinomanometry. Eur Respir J 1998; 11: 720-725.

15. Lorino AM, Lofaso F, Duizabo D, et al. Respiratory resistive impedance as an index of airway obstruction during nasal continuous positive airway pressure titration. Am J Respir Crit Care Med 1998; 158: 1465-1470.

16. Liistro G, Stanescu D, Dooms G, Rodenstein R, Veriter C. Head position modifies upper airway resistance in men. $J$ Appl Physiol 1988; 64: 1285-1288.

17. Lowe A, Fleetham J, Ryan F, Mathews B. Effects of a mandibular repositionning appliance used in the treatment of obstructive sleep apnea on tongue muscle activity. In: Issa FG, Suratt PM, Remmers JE, eds. Sleep and Respiration. New York, Wiley-Liss, 1990; 395-405.

18. Rowley JA, Permutt S, Willey S, Smith PL, Schwartz AR. Effect of tracheal and tongue displacement on upper airway airflow dynamics. J Appl Physiol 1996; 80: 21712178.

19. Widdicombe JG. Vagal reflexes in the airways. In: Kaliner AK, Barnes PJ, eds. The Airways: Neural Control in Health and Disease. New York, Dekker, 1988; 187202. 